\title{
Are neuroendocrine negative small cell lung cancer and large cell neuroendocrine carcinoma with WT RB1 two faces of the same entity?
}

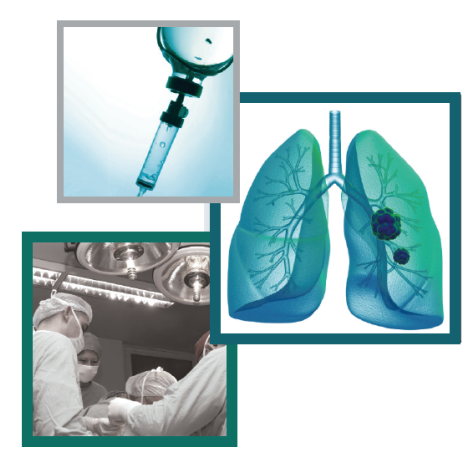

\author{
Dmitriy Sonkin*,1 (D), Anish Thomas ${ }^{2}$ \& Beverly A Teicher $* *, 1$ \\ ${ }^{1}$ National Cancer Institute, Division of Cancer Treatment \& Diagnosis, Rockville, MD 20850, USA \\ ${ }^{2}$ National Cancer Institute, Center for Cancer Research, Bethesda, MD 20892, USA \\ *Author for correspondence: dmitriy.sonkin@nih.gov \\ **Author for correspondence: beverly.teicher@nih.gov
}

\section{Practice points}

- Similarity between some subtypes of small cell lung cancer (SCLC) and large cell neuroendocrine carcinoma (LCNEC) can be even higher than between SCLC and LCNEC in general.

- Neuroendocrine markers negative/low RB1 WT SCLC-Y subtype and neuroendocrine markers negative/low WT RB1, WT KEAP1, WT STK11 LCNEC subtype are highly similar and might be in fact the same entity.

- Unanimous agreement between expert pathologists is around $50 \%$ in differentiating between SCLC and LCNEC, indicating high level of diagnostic variability.

- Patients with neuroendocrine markers negative/low WT RB1, WT KEAP1, WT STK11 LCNEC subtype might be candidates for clinical trial-based treatment with CDK4/6 inhibitors.

- Lung anatomic locations could be important information to record along with genomic information and neuroendocrine markers staining.

- About $10 \%$ of tumors diagnosed as SCLC are RB1 WT, such tumors may have sensitivity to treatments which are not applicable to majority of SCLC with inactivated RB1, therefore, confirming RB1 status should be an important consideration.

Until recently, small cell lung cancer (SCLC) was described as SCLC and SCLC variant, based upon cellular morphology and loss of neuroendocrine markers in the SCLC variant. However, based on recent research advances, driven in part by the increase in comprehensive genomic data, it has become clear that there are multiple SCLC subtypes including an ASCL1 and NEUROD1 low, YAP1 high (SCLC-Y) subtype enriched for WT RB1. Comparing morphological and other features of this SCLC subtype to neuroendocrine negative RB1, KEAP1, STK11 WT LCNEC raises a number of important questions with diagnostic and therapeutic implications.

First draft submitted: 30 May 2019; Accepted for publication: 11 June 2019; Published online: 21 August 2019

Keywords: CDK4/6 inhibitor • LCNEC • neuroendocrine negative $\bullet$ RB1 WT • SCLC

Small cell lung cancer (SCLC) is an aggressive form of lung cancer with limited therapeutic options, a very high mortality rate and is characterized, in most cases, by neuroendocrine features. SCLC accounts for approximately $15 \%$ of lung cancers. The majority of SCLC are genetically characterized by bi-allelic inactivation of RB1 ( 90\%) and TP53 ( 98\%) tumor suppressor genes [1-6]. The prevailing hypothesis is that inactivation of RB1 in SCLC leads to increase in cellular proliferation due to loss of cell cycle control and inactivation of TP53 prevents oncogeneinduced senescence. SCLC diagnosis is commonly based on morphological features of biopsy or cytology samples. A panel of neuroendocrine markers (CHGA, NCAM1, SYP) may also be utilized [7,8]. In a noticeable fraction of cases, SCLC is present along with other lung cancer subtype(s) such as: large cell neuroendocrine carcinoma (LCNEC), large cell carcinoma (LCC), adenocarcinoma and/or squamous cell carcinoma [9]. 


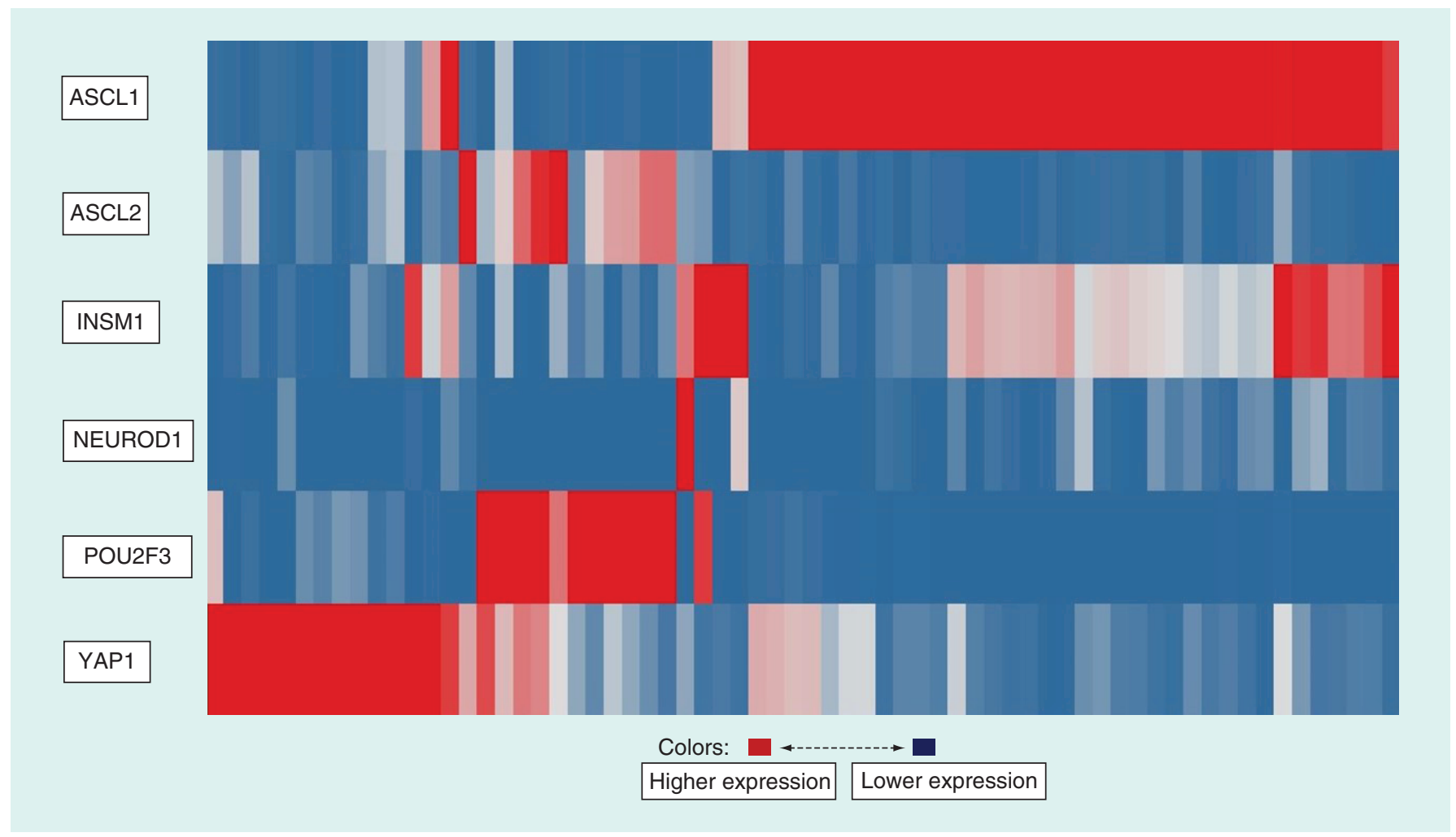

Figure 1. Gene expression clustering of large cell neuroendocrine carcinoma by key 'small cell lung cancer' transcription factors.

Currently, SCLC is divided into four subtypes: ASCL1 high (SCLC-A), NEUROD1 high (SCLC-N), POU2F3 high (SCLC-P) and YAP1 high (SCLC-Y) subtype enriched for WT RB1 [10-13]. SCLC-Y subtype has low or absent expression of ASCL1, NEUROD1 and other neuroendocrine markers and accounts for approximately 5$10 \%$ SCLC tumors $[11,12]$. The SCLC-Y subtype is enriched for CCND1 amplification and CDKN2A inactivation, these alterations may play similar role to RB1 inactivation resulting in cell cycle control defects [11].

LCNEC is a relatively rare lung cancer, accounting for approximately $3 \%$ of lung cancer cases. In general, LCNEC is characterized by neuroendocrine morphology and markers; similarly to SCLC, patients with LCNEC have a poor prognosis [14]. About 90\% of LCNEC have bi-allelic TP53 inactivation, 40\% have bi-allelic RB1 inactivation and $\sim 40 \%$ have KEAP1 and/or bi-allelic STK11 inactivation; bi-allelic RB1 inactivation is generally mutually exclusive with KEAP1/STK11 bi-allelic inactivation [15,16]. From genetic alterations, prospective LCNEC with bi-allelic RB1 and TP53 inactivation are considered 'SCLC like' and LCNEC with KEAP1/STK11 bi-allelic inactivation are considered 'NSCLC like'. About 4-8\% of LCNEC are ASCL1 and NEUROD1 low with WT RB1, WT KEAP1, WT STK11 and bi-allelic TP53 inactivation. It is interesting to note that clustering gene expression LCNEC data from George et al. using key 'SCLC' transcription factors results in the gene expression pattern shown in Figure 1 which is very similar to one observed in SCLC [10,16].

Thus, there are noticeable genetics, genomics and phenotypical similarities between the RB1 WT SCLC-Y subtype and the WT RB1, WT KEAP1, WT STK11 LCNEC subtype, where each subtype displays low or absent expression of ASCL1 and NEUROD1. In order to provide a more detailed background for comparison, some of the key features of neuroendocrine negative (NE-) SCLC are listed in Table 1. For comparison purposes, features of classical neuroendocrine positive (NE+) SCLC are also listed. Properties of LCNEC listed in Table 1 are generic and not necessarily specific to neuroendocrine negative (NE-) LCNEC subtype with WT RB1, WT KEAP1, WT STK11; it is possible that such LCNEC subtype has morphological properties even more similar to neuroendocrine negative (NE-) SCLC.

In general, SCLC are mostly located in central lung location and LCNEC in peripheral or midzone lung location [17-19]. A recent study from Zhou et al. suggests the possibility of differences between LCNEC in pe- 
Table 1. Morphological properties of large cell neuroendocrine carcinoma, neuroendocrine negative small cell lung cancer and classical neuroendocrine positive small cell lung cancer.

\begin{tabular}{|llll|}
\hline Feature & LCNEC & SCLC NE- & SCLC NE+ \\
\hline Mostly smokers & Yes & Yes & Yes \\
\hline Median age & $\sim 67$ years & $\sim 65$ years & $\sim 65$ years \\
\hline Cell size & Typical of NSCLC & Larger than classical SCLC & Small \\
\hline Cytoplasm & Abundant & More plentiful than classical SCLC & Scarce \\
\hline Nucleoli & Prominent & Prominent & High \\
\hline Mitotic rate & High & More uniform & High \\
\hline Nuclear chromatin & Less uniform & Less distinct & Lore uniform \\
\hline Cell borders & More distinct & \\
\hline
\end{tabular}

LCNEC: Large cell neuroendocrine carcinoma; NE: Neuroendocrine; NSCLC: Non-small-cell lung carcinoma; SCLC: Small cell lung cancer.

ripheral versus central locations [20]. Unfortunately, data regarding tumor location in the lung is limited for the neuroendocrine-negative SCLC subtype and for neuroendocrine-negative LCNEC with WT RB1, KEAP1, STK1 1 .

There is currently no definitive marker(s) for LCNEC and diagnosis is based on exclusion. In part due to this and significant variabilities in LCNEC and SCLC phenotypes, there is limited agreement among pathologists on the LCNEC diagnosis. Ha et al. indicated that, among five expert thoracic pathologists, unanimous agreement in the diagnosis of LCNEC vs SCLC was achieved in only $40 \%$ of cases, other studies indicate similar levels of unanimous agreement between expert pathologists in SCLC and LCNEC diagnosis [21-23]. As can be seen from Table 1, neuroendocrine negative SCLC and LCNEC have a number of similarities and potential differences that are rather subtle, which makes it even more difficult to achieve agreement between pathologists.

In the Nicholson et al. study, out of 100 surgical biopsies or resections with a diagnosis of SCLC, 28 showed evidence of an NSCLC component in addition to SCLC, with LCC being the largest mixture component present in 16 cases of combined SCLC. Out of these 16 SCLC/LCC combined cases, 6 had LCNEC subtype of LCC [24]. In most instances of NSCLC combined with SCLC there is strong evidence of clonality between SCLC and NSCLC components, likely indicating a common precursor cell in such cases [25-29]. Pulmonary neuroendocrine cells (PNECs) and perhaps common pulmonary stem cells are potential cells of origin for SCLC and LCNEC [30-32].

Keeping in mind a likely common cell of origin and very similar genetics, genomics and morphology of the RB1 WT SCLC-Y subtype and the WT RB1, WT KEAP1, WT STK11 LCNEC subtype with low or absent expression of ASCL1 and NEUROD1, it is tempting to suggest that perhaps these two lung cancer subtypes are in-fact two faces of the same entity. If this is indeed the case, there are important diagnostic and therapeutic implications. From a diagnostic prospective, it should be important to note that such tumors share SCLC and LCNEC properties. Such acknowledgment has a potential therapeutic implication in relation to CDK4/6 inhibitors. Due to their lack of functional RB1, the clear majority of SCLC models are insensitive to the CDK4/6 inhibitors [33-36]. In contrast, some SCLC models with functional RB1 are sensitive to the CDK4/6 inhibitors [11,12,36,37]. Following these preclinical studies, a clinical trial of a CDK4/6 inhibitor in RB1 WT SCLC is being developed. Based on the discussion in this perspective, patients diagnosed with LCNEC subtype with WT RB1, WT KEAP1, WT STK11 and loss of neuroendocrine markers may benefit from being considered eligible for such clinical trial.

\section{Future perspective}

We hope that analysis presented in this article would help to increase awareness about highly similar subtypes of SCLC and LCNEC and potential clinical implications which might became even more relevant in future. We also hope our work would help to rejuvenate efforts on collecting information on lung anatomic locations of SCLC, LCNEC (and perhaps other lung tumors) in addition to genomic information and neuroendocrine markers staining. It is possible that paying attention to lung location such as: peripheral, midzone or central along with genomic information and neuroendocrine status may help to better characterize lung malignancies.

\section{Acknowledgments}

The views expressed in this article are the personal opinions of the authors and do not necessarily reflect policy of the US National Cancer Institute. 
Financial \& competing interests disclosure

This article is a US Government work and is in the public domain in the USA. The authors have no other relevant affiliations or financial involvement with any organization or entity with a financial interest in or financial conflict with the subject matter or materials discussed in the manuscript apart from those disclosed.

No writing assistance was utilized in the production of this manuscript.

Open access

This work is licensed under theAttribution-NonCommercial-NoDerivatives 4.0 Unported License. To view a copy of this license, visit http://creativecommons.org/licenses/by-nc-nd/4.0/

\section{References}

Papers of special note have been highlighted as: $\bullet$ of interest;

1. George J, Lim JS, Jang SJ et al. Comprehensive genomic profiles of small cell lung cancer. Nature 524(7563), 47-53 (2015).

- Provides comprehensive genomic profiles of small cell lung cancer (SCLC), including exome and RNA sequencing.

2. van Meerbeeck JP, Fennell DA, De Ruysscher DKM. Small-cell lung cancer. Lancet 378(9804), 1741-1755 (2011).

3. Weinberg RA. The retinoblastoma protein and cell cycle control. Cell 81(3), 323-330 (1995).

4. Knudson AG. Mutation and cancer: statistical study of retinoblastoma. Proc. Natl Acad. Sci. USA 68(4), 820-823 (1971).

5. Goodrich DW. The retinoblastoma tumor-suppressor gene, the exception that proves the rule. Oncogene 25(38), 5233-5243 (2006).

6. Levine AJ, Oren M. The first 30 years of p53: growing ever more complex. Nat. Rev. Cancer 9(10), 749-758 (2009).

7. Rekhtman N. Neuroendocrine tumors of the lung: an update. Arch. Pathol. Lab. Med. 134(11), 1628-1638 (2010).

8. Travis WD. Update on small cell carcinoma and its differentiation from squamous cell carcinoma and other non-small cell carcinomas. Mod. Pathol. 25, S18-S30 (2012).

9. Nicholson SA, Beasley MB, Brambilla E et al. Small cell lung carcinoma (SCLC): a clinicopathologic study of 100 cases with surgical specimens. Am. J. Surg. Pathol. 26(9), 1184-1197 (2002).

10. Rudin CM, Poirier JT, Byers LA et al. Molecular subtypes of small cell lung cancer: a synthesis of human and mouse model data. Nat. Rev. Cancer 19, 289-297 (2019).

- Reviews current knowledge on SCLC and proposes genomics-based classification.

11. Sonkin D, Vural S, Thomas A, Teicher BA. Neuroendocrine negative SCLC is mostly RB1 WT and may be sensitive to CDK4/6 inhibition. bioRxiv. doi: 10.1101/516351 (2019) (Epub ahead of print).

- Provides detailed genomic analysis of SCLC subtype with high YAP1 expression, loss of neuroendocrine markers and WT RB1.

12. McColl K, Wildey G, Sakre N et al. Reciprocal expression of INSM1 and YAP1 defines subgroups in small cell lung cancer. Oncotarget 8(43), 73745-73756 (2017).

- Describes SCLC subtype with high YAP1 expression, loss of neuroendocrine markers and enrichment for WT RB1.

13. Huang Y-H, Klingbeil O, He X-Y et al. POU2F3 is a master regulator of a tuft cell-like variant of small cell lung cancer. Genes Dev. 32(13-14), 915-928 (2018).

14. Fasano M, Della Corte CM, Papaccio F, Ciardiello F, Morgillo F. Pulmonary large-cell neuroendocrine carcinoma: from epidemiology to therapy. J. Thorac. Oncol. 10(8), 1133-1141 (2015).

15. Rekhtman N, Pietanza MC, Hellmann MD et al. Next-generation sequencing of pulmonary large cell neuroendocrine carcinoma reveals small cell carcinoma-like and non-small cell carcinoma-like subsets. Clin. Cancer Res. 22(14), 3618-3629 (2016).

16. George J, Walter V, Peifer $\mathrm{M}$ et al. Integrative genomic profiling of large-cell neuroendocrine carcinomas reveals distinct subtypes of high-grade neuroendocrine lung tumors. Nat. Commun. 9(1), 1048 (2018).

- Provides comprehensive genomic profiles of large cell neuroendocrine carcinoma (LCNEC) and outlines existence of distinct subtypes.

17. Oshiro $\mathrm{Y}$, Kusumoto M, Matsuno $\mathrm{Y}$ et al. CT findings of surgically resected large cell neuroendocrine carcinoma of the lung in 38 patients. AJR Am. J. Roentgenol. 182(1), 87-91 (2004).

18. Jung KJ, Lee KS, Han J et al. Large cell neuroendocrine carcinoma of the lung: clinical, CT, and pathologic findings in 11 patients. $J$. Thorac. Imaging 16(3), 156-162 (2001).

19. Rosado-de-Christenson ML, Templeton PA, Moran CA. Bronchogenic carcinoma: radiologic-pathologic correlation. RadioGraphics 14(2), 429-446 (1994).

20. Zhou F, Hou L, Ding T et al. Distinct clinicopathologic features, genomic characteristics and survival of central and peripheral pulmonary large cell neuroendocrine carcinoma: From different origin cells? Lung Cancer Amst. Neth. 116, 30-37 (2018).

21. Travis WD, Gal AA, Colby TV, Klimstra DS, Falk R, Koss MN. Reproducibility of neuroendocrine lung tumor classification. Hum. Pathol. 29(3), 272-279 (1998). 
22. den Bakker MA, Willemsen S, Grünberg $\mathrm{K}$ et al. Small cell carcinoma of the lung and large cell neuroendocrine carcinoma interobserver variability. Histopathology 56(3), 356-363 (2010).

- Describes challenges in differentiating between SCLC and LCNEC and corresponding high diagnostic variability.

23. Ha SY, Han J, Kim W-S, Suh BS, Roh MS. Interobserver variability in diagnosing high-grade neuroendocrine carcinoma of the lung and comparing it with the morphometric analysis. Korean J. Pathol. 46(1), 42-47 (2012).

24. Nicholson SA, Beasley MB, Brambilla E et al. Small cell lung carcinoma (SCLC): a clinicopathologic study of 100 cases with surgical specimens. Am. J. Surg. Pathol. 26(9), 1184-1197 (2002).

25. D’Adda T, Pelosi G, Lagrasta C et al. Genetic alterations in combined neuroendocrine neoplasms of the lung. Mod. Pathol. 21(4), 414-422 (2008).

26. Fellegara G, D'Adda T, Pilato FP et al. Genetics of a combined lung small cell carcinoma and large cell neuroendocrine carcinoma with adenocarcinoma. Virchows Arch. 453(1), 107-115 (2008).

27. Huang J, Behrens C, Wistuba II, Gazdar AF, Jagirdar J. Clonality of combined tumors. Arch. Pathol. Lab. Med. 126(4), 437-441 (2002).

28. Murase T, Takino H, Shimizu S et al. Clonality analysis of different histological components in combined small cell and non-small cell carcinoma of the lung. Hum. Pathol. 34(11), 1178-1184 (2003).

29. Wagner PL, Kitabayashi N, Chen Y-T, Saqi A. Combined small cell lung carcinomas: genotypic and immunophenotypic analysis of the separate morphologic components. Am. J. Clin. Pathol. 131(3), 376-382 (2009).

30. Pathology and genetics of tumours of the lung, pleura, thymus and heart. World Health Organization classification of tumors. Travis WD, Brambilla E, Müller-Hermelink HK, Harris CC (Eds.), IARC Press, Lyon, France (2004).

31. Swarts DRA, Ramaekers FCS, Speel E-JM. Molecular and cellular biology of neuroendocrine lung tumors: evidence for separate biological entities. Biochim. Biophys. Acta. 1826(2), 255-271 (2012).

32. Sutherland KD, Berns A. Cell of origin of lung cancer. Mol. Oncol. 4(5), 397-403 (2010).

33. O’Leary B, Finn RS, Turner NC. Treating cancer with selective CDK4/6 inhibitors. Nat. Rev. Clin. Oncol. 13(7), 417-430 (2016).

34. Baughn LB, Di Liberto M, Wu K et al. A novel orally active small molecule potently induces G1 arrest in primary myeloma cells and prevents tumor growth by specific inhibition of cyclin-dependent kinase 4/6. Cancer Res. 66(15), 7661-7667 (2006).

35. Fry DW, Harvey PJ, Keller PR et al. Specific inhibition of cyclin-dependent kinase 4/6 by PD 0332991 and associated antitumor activity in human tumor xenografts. Mol. Cancer Ther. 3(11), 1427-1438 (2004).

36. Polley E, Kunkel M, Evans D et al. Small cell lung cancer screen of oncology drugs, investigational agents, and gene and microRNA expression. J. Natl Cancer Inst. 108(10) (2016).

37. Barretina J, Caponigro G, Stransky N et al. The Cancer Cell Line Encyclopedia enables predictive modelling of anticancer drug sensitivity. Nature 483(7391), 603-607 (2012). 